\title{
Evaluation of non-specific effects of infant immunizations on early infant mortality in a southern Indian population
}

\author{
Lawrence H. Moulton', Lakshmi Rahmathullah², Neal A. Halsey', R. D. Thulasiraj ${ }^{3}$, Joanne Katz' \\ and James M. Tielsch' \\ 1 Department of International Health, Johns Hopkins Bloomberg School of Public Health, Baltimore, MD, USA \\ 2 Aravind Centre for Women, Children and Community Health, Madurai, Tamil Nadu, India \\ 3 Lions-Aravind Institute for Community Ophthalmology, Madurai, Tamil Nadu, India
}

Summary OBJEctive The aim of this study was to assess the relationship between receipt of routine childhood immunizations and infant mortality before 6 months of age.

METHODS This was an observational study of 10274 infants, in a randomized trial of vitamin A supplementation, who received the study dose and survived to at least 1 week of age. The primary outcome was mortality before 6 months of age, analysed in Cox regression models as a function of vaccine receipt and gender.

RESUlTs Receipt of Bacille Calmette Guerin (BCG) or diphtheria, tetanus, polio (DTP) vaccine was associated with significant reductions of one-half to two-thirds of mortality hazards; among girls, those who received both BCG and DTP experienced higher mortality than those who received only one of the two vaccines (hazards ratio $2.4 ; 95 \%$ confidence interval $1.2-5.0$ ).

CONCLUSION The reduced mortality rate associated with receipt of BCG or DTP may be due to both biological and selection factors; the analyses regarding the combined effect of these vaccines and gender need to be replicated in other settings.

keywords vaccines, infant mortality, vitamin A, BCG, DTP

\section{Introduction}

Recent studies have investigated the relationship between immunization and survival in countries with relatively high background mortality rates (Kristensen et al. 2000; Aaby et al. 2002). Although the possible role of measles vaccine in lowering mortality in children without measles has long been of interest (Aaby et al. 1995, 1996; Kabir et al. 2003), non-specific effects of other vaccines on mortality have received little attention. In a study of children under 2 years of age in Guinea-Bissau, Kristensen et al. (2000) found immunization with Bacille Calmette Guerin (BCG) vaccine to be associated with lower mortality, but stated that oral polio vaccine (OPV) and diphtheria, tetanus, polio (DTP) vaccines were associated with higher mortality. More recently, it has been suggested that this effect may be gender-specific, existing primarily among girls (Aaby et al. 2002).

The World Health Organization (WHO) organized a workshop at which hypotheses were drawn up for further investigation (WHO 2001). In particular, it was recommended that participants focus on mortality during the first 2 years of life as a function of early receipt of BCG vaccine and initiation of DTP immunization within the first 6 months. Guidelines also focused on the effect of gender, and on vaccine/gender interactions. We address some of these in the context of a randomized field trial of vitamin A supplementation. The focus is on relating timing of BCG and DTP vaccine receipt to mortality from 1 week to 6 months of age, with emphasis on gender differentials.

\section{Materials and methods}

Study population background

The Vitamin A Supplementation in Newborns Study (VASIN) was the result of a collaboration between the Aravind Centre for Women, Children and Community Health, Tamil Nadu and the Department of International Health, Johns Hopkins Bloomberg School of Public Health, Baltimore. This study followed up infants from birth to 6 months of age, and reported a $22 \%$ reduction in total mortality for those who received vitamin A near the 
L. H. Moulton et al. Infant immunizations and early infant mortality in India

time of birth (for more details, see Rahmathullah et al. 2003).

\section{Data collection}

All live births between 12 August 1998 and 14 February 2002 in two rural blocks of Tamil Nadu were considered for the study. On each of the first 2 days of life, infants were given a dose of 24000 IU of vitamin A or placebo. Every 2 weeks, infants were visited to record information on vital status, morbidity, immunization, clinic visits and other health events. At each follow-up visit, the mother was queried regarding immunizations during the previous 2 weeks. If the mother stated that a vaccination had occurred in the past 2 weeks, but could not recall the type of vaccine, the field worker checked the child's immunization card if it was available in the house. For each day with no immunizations, a missing code was entered, distinguishing it from the situation 'received but doesn't know which ones,' which received a different code. If an infant was reported dead, a verbal autopsy was conducted. If a baby was not followed up for the full 6 months, but was alive at the last visit, we designated the infant as being lost to follow-up at the date of that visit.

\section{Mortality outcome}

All analyses pertain to deaths among infants who were given a study dose and survived to 1 week of age. We considered receipt of a study dose as a positive indication of the infant's enrolment status, and of high likelihood of being followed up as part of the study. We eliminated deaths in the first week, thinking they could not be due to non-specific effects of BCG vaccine, and that the high mortality in that first week might cloud relevant associations. The verbal autopsy data would not permit diseasespecific determinations, and thus we have analysed allcause mortality. It may be that DTP vaccine directly prevented a few deaths that otherwise would have occurred as a consequence of diphtheria, tetanus, or pertussis, but we expect that in this population, most of any mortality reduction associated with immunization would be due to non-specific effects of the vaccine's stimulation of the immune system.

\section{Statistical methods}

To handle the high degree of overlap in time of immunizations and deaths, time-varying covariates were employed in Cox proportional hazards regression models. The usual strong relationship of mortality to infant age was adjusted for automatically by aligning histories at birth, i.e. infant age was used as the timeline for the Cox models. Timevarying binary covariates were constructed for the initial receipt of each vaccine.

Other possibly confounding covariates were incorporated in some models. The familial and household characteristics in Table 2 were used to form propensity scores (Rosenbaum \& Rubin 1983) via logistic regression models, for likelihood of being immunized with BCG, OPV, or DTP vaccine. In each model, two dummy variables indicating those most (top 10\%) and least (bottom 10\%) likely to be immunized were created.

Censoring was at the earliest of: death, 6 months of age, loss to follow-up, and receipt of first unknown vaccine. We investigated the role of gender both as a main effect and as an effect modifier of vaccine effects. Because our data were from a study of vitamin A supplementation with demonstrated effects, we conducted exploratory analyses of the potential impact of receipt of vitamin A on our study results.

Tabled results of model fits are given in terms of hazards ratios (HR) comparing vaccinated to unvaccinated infants. A ratio less than unity indicates improved survival with vaccine receipt. To construct Figure 1, each infant was classified into one of four vaccine categories as of 60 days of age. In preparation for the WHO workshop (WHO 2001), BCG vaccine receipt by 1 month of age had been a focus of some analyses, and median ages of receipt were close to 30 days for BCG and 60 days for DTP; thus, these two cut-off points were employed in order to produce the curves. Note that infants who receive BCG after 30 days or DTP after 60 days remain in the categories of no receipt, whereas the Cox models account for changing vaccination status.

The regression models were fit using SAS PROC PHREG (v.8.0; SAS Inc., Cary, NC, USA); all other analyses were performed with Stata (v.7; StataCorp, College Station, TX, USA). All $P$-values are based on two-sided alternative hypotheses.

\section{Results}

\section{Basic characteristics}

Of the 13294 infants born to enrolled women in the study area during the study period, 11619 infants were born alive and enrolled, and 10274 were alive as of 7 days after birth and had received the study dose of either vitamin A or placebo. There were 208 recorded deaths; of the remaining 10066 infants, $8845(83 \%)$ were followed up to at least 90 days of age. Of the 2884 who were not followed up to 6 months of age, 629 were administratively censored at study end, having been enrolled $<6$ months before the study ended. 
L. H. Moulton et al. Infant immunizations and early infant mortality in India

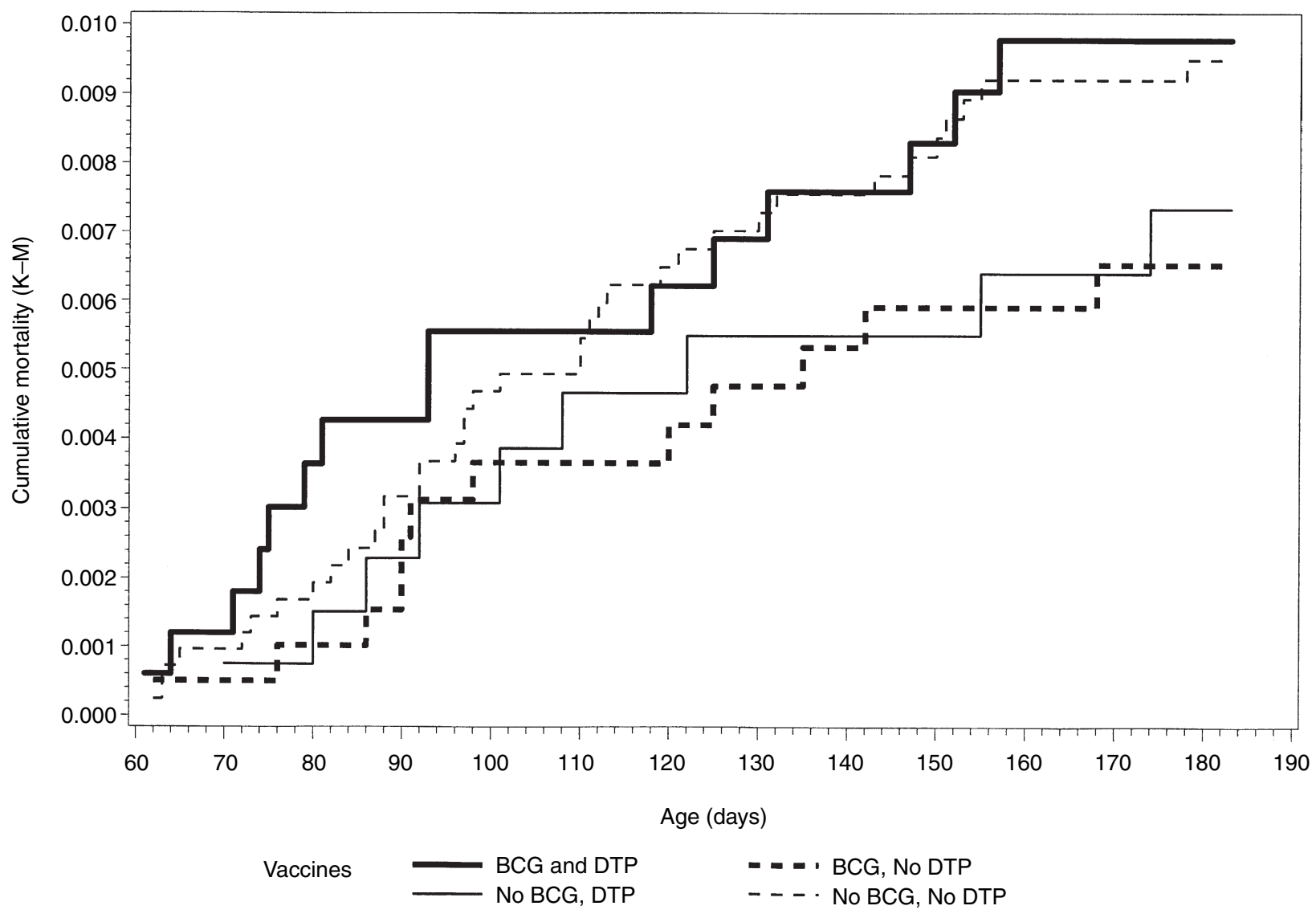

Figure I Kaplan-Meier cumulative mortality curves by immunization status as of 30 days (BCG) and 60 days (DPT); 1998-2002, Tamil Nadu, India.

During the first 6 months of life, the standard immunization schedule in India is: BCG (0-12 months), DTP $(6$, 10, 14 weeks), OPV (birth, 6, 10, 14 weeks). Of the $59.1 \%$ of infants who received a BCG dose before 6 months, half were immunized by 2.7 weeks of age, while the median age at first receipt of the $82.8 \%$ immunized with OPV was 6.7 weeks (Table 1). Only $65.9 \%$ ever received DTP; of those who did, the median age at first receipt was
8.9 weeks. Although $44.2 \%$ received at least one dose of each of the vaccines (BCG, DTP, OPV), only $12.7 \%$ received at least one BCG, three DTP, and three OPV immunizations by 6 months of age. Less than $2 \%$ of the recorded immunizations were of an unknown type.

Vaccine coverage was similar across gender (Table 2). There was a tendency for infants with higher birthweight to have greater coverage. Infants born in families with

Table I Vaccine receipt by 6 months of age and distribution of age of receipt among those immunized; dosed study participants; 1998-2002, Tamil Nadu, India

\begin{tabular}{llllllll}
\hline & BCG & OPV1 & OPV2 & OPV3 & DTP1 & DTP2 & DTP3 \\
\hline $\begin{array}{lllllll}\text { Percent }(n) \text { ever received by 6 months } \\
\text { Percentile }\end{array}$ & $\begin{array}{l}59.1(6069) \\
\text { Weeks of age }\end{array}$ & $82.8(8511)$ & $65.2(6700)$ & $45.7(4697)$ & $65.9(6775)$ & $39.4(4045)$ & $17.4(1783)$ \\
10 & 0.4 & 1.3 & 6.1 & 8.6 & 6.4 & 11.0 & 15.3 \\
50 & 2.7 & 6.7 & 11.9 & 15.6 & 8.9 & 14.6 & 18.6 \\
90 & 9.9 & 16 & 20.6 & 22.7 & 17.3 & 20.9 & 23.0 \\
\cline { 2 - 8 } & & & & & & & \\
\hline
\end{tabular}


L. H. Moulton et al. Infant immunizations and early infant mortality in India

\begin{tabular}{|c|c|c|c|c|c|}
\hline \multirow[b]{2}{*}{ Characteristics } & \multirow[b]{2}{*}{$n$} & \multicolumn{4}{|c|}{ Receiving vaccine } \\
\hline & & $\operatorname{BCG}[\%(n)]$ & OPV1 $[\%(n)]$ & DTP1 $[\%(n)]$ & $\operatorname{Died}[\%(n)]$ \\
\hline \multicolumn{6}{|l|}{ Personal } \\
\hline \multicolumn{6}{|l|}{ Gender } \\
\hline Female & 5005 & $59(2936)$ & $82(4120)$ & $66(3293)$ & $2.2(110)$ \\
\hline Male & 5269 & $59(3133)$ & $83(4391)$ & $66(3482)$ & $1.9(98)$ \\
\hline \multicolumn{6}{|c|}{ Birthweight (g) } \\
\hline$\geq 2500$ & 6880 & $60(4155)$ & $84(5755)$ & $67(4578)$ & $1.2(85)$ \\
\hline$<2500$ & 3394 & $56(1914)$ & $81(2756)$ & 65 (2197) & $3.6(123)$ \\
\hline \multicolumn{6}{|c|}{ Mother's prior live births } \\
\hline$<3$ & 8858 & $60(5281)$ & $82(7305)$ & 66 (5839) & 1.8 \\
\hline$\geq 3$ & 1416 & $56(788)$ & $85(1206)$ & $66(936)$ & $3.6(51)$ \\
\hline \multicolumn{6}{|c|}{ Household/familial } \\
\hline \multicolumn{6}{|c|}{ Wood fuel } \\
\hline Yes & 9556 & $58(5539)$ & $83(7921)$ & $66(6268)$ & $2.1(201)$ \\
\hline No & 718 & $74(530)$ & $82(590)$ & $71(507)$ & $1.0(7)$ \\
\hline \multicolumn{6}{|l|}{ Hard roof } \\
\hline Yes & 6965 & $63(4358)$ & $84(5853)$ & $68(4767)$ & $1.9(134)$ \\
\hline No & 3309 & $52(1711)$ & $80(2658)$ & $61(2008)$ & $2.2(74)$ \\
\hline \multicolumn{6}{|c|}{ Maternal education } \\
\hline Yes & 4758 & $63(3612)$ & $83(4758)$ & 69 (3942) & 1.7 (97) \\
\hline No & 3753 & $54(2457)$ & $82(3753)$ & $62(2834)$ & $2.4(111)$ \\
\hline \multicolumn{6}{|c|}{ Have electricity } \\
\hline Yes & 5807 & $64(3709)$ & $84(4884)$ & $69(3987)$ & $1.8(102)$ \\
\hline No & 4467 & $53(2360)$ & $81(3627)$ & $62(2788)$ & $2.4(106)$ \\
\hline \multicolumn{6}{|l|}{ Have TV } \\
\hline Yes & 1864 & $70(1305)$ & $84(1563)$ & $72(1339)$ & $1.6(30)$ \\
\hline No & 8410 & $57(4764)$ & $83(6948)$ & $65(5436)$ & $2.1(178)$ \\
\hline \multicolumn{6}{|c|}{ Own or lease land } \\
\hline Yes & 5258 & $57(2981)$ & $83(4356)$ & $63(3320)$ & $2.3(121)$ \\
\hline No & 5016 & $62(3088)$ & $83(4155)$ & $69(3455)$ & $1.7(87)$ \\
\hline \multicolumn{6}{|l|}{ Own cattle } \\
\hline Yes & 2854 & $53(1506)$ & $82(2329)$ & $61(1745)$ & $2.6(73)$ \\
\hline No & 7420 & $62(4563)$ & $83(6182)$ & $68(5030)$ & $1.8(135)$ \\
\hline
\end{tabular}

Table 2 Characteristics of dosed participants and \% ages ever receiving vaccines or dying by 6 months of age; 1998-2002, Tamil Nadu, India higher socioeconomic status (SES) as measured by having a hard roof for their home, electricity, a television set, or some maternal education also had higher vaccine coverage. Using wood fuel, owning cattle or owning or leasing land - proxies for farming or a more rural existence indicated lower coverage. Infant mortality followed these patterns, with a higher proportion of infants dying in those SES categories associated with lower vaccine coverage.

The distribution of deaths according to immunization status, gender, and age is presented in Table 3. It is provided for descriptive purposes; care must be taken in its interpretation. For example, among those who did not receive BCG, the mortality for infants with DTP in the first 60 days is lower than for no DTP, as the ones who have received DTP have survived long enough to receive it, outliving the perinatal period of higher mortality risk.

\section{Survival analyses}

Univariate results of the time-varying immunization models are displayed as models A-F in Table 4. Depending on the model, there were $25-32 \%$ reductions in mortality. Statistical significance increased slightly for OPV in the adjusted models, and decreased slightly for BCG and DTP. Placing two or three of the vaccine variables in the same model had little effect on their individual HR (models G and $\mathrm{H}$ ).

Focusing on the combined use of BCG and DTP, the rest of the models in Table 4 include three dummy variables to track the four potential categories of vaccine status at any moment in an infant's life. Among those who did not receive BCG, receipt of DTP vaccine was associated with a $56 \%$ reduction [95\% confidence interval (CI): 21-76; $P=0.007]$ in mortality (model I). The same mortality 


\section{H. Moulton et al. Infant immunizations and early infant mortality in India}

Table 3 Mortality by gender, age, and BCG-DPT immunization status; 1998-2002, Tamil Nadu, India

\begin{tabular}{|c|c|c|c|c|c|c|}
\hline \multirow[b]{2}{*}{ Immunization status by age } & \multicolumn{3}{|l|}{ Females } & \multicolumn{3}{|l|}{ Males } \\
\hline & Deaths & Infant-years & Rate/100 infant-years & Deaths & Infant-years & Rate/100 infant-years \\
\hline \multicolumn{7}{|l|}{1 week to 59 days } \\
\hline No BCG; no DPT & 62 & 400 & 15.4 & 49 & 418 & 11.7 \\
\hline BCG; no DPT & 7 & 242 & 2.9 & 17 & 265 & 6.4 \\
\hline No BCG; DPT & 0 & 17 & 0.0 & 0 & 17 & 0.0 \\
\hline BCG; DPT & 0 & 29 & 0.0 & 0 & 32 & 0.0 \\
\hline \multicolumn{7}{|l|}{60 days to 6 months } \\
\hline No BCG; no DPT & 11 & 287 & 3.8 & 9 & 307 & 2.9 \\
\hline BCG; no DPT & 8 & 224 & 3.6 & 6 & 239 & 2.5 \\
\hline No BCG; DPT & 6 & 273 & 2.2 & 7 & 276 & 2.5 \\
\hline BCG; DPT & 16 & 552 & 2.9 & 10 & 613 & 1.6 \\
\hline
\end{tabular}

hazard was seen for those who, conversely, received BCG but not DTP. Receipt of both vaccines, however, was only slightly beneficial relative to having had neither (rate reduction: $28 \%, 95 \% \mathrm{CI}:-20,57 ; P=0.20)$. The difference in the effect associated with DTP, from risk reduction in the absence of BCG to risk increase following BCG receipt $(0.44 / 1$ to $0.72 / 0.44)$, was highly statistically significant $(P=0.0007$ for interaction). Comparing the risk of both BCG and DTP with the mean risk of the singlevaccine categories, we get a HR of $1.6(P=0.066)$.

Figure 1 shows a similar pattern, with higher mortality risks for infants that are categorized as having neither BCG nor DTP vaccine, or both.

Fitting the same model separately for males and females, we found a negligible interaction (with $P=0.25$ ) among the males (model J), with their hazard rate remaining about the same when both vaccines were received as when only one had been received. The interaction was accentuated, however, among females (model K), and females with two vaccines were more than twice as likely to die as females with only one vaccine $(P=0.0148)$. In a reparameterized model fit to all the data, the difference between the two genders' BCG/DTP interaction terms was statistically significant $(P=0.03)$. Adjusting the model for females by the addition of OPV, the propensity score variables for BCG, and those covariates with $P<0.20$ in models $\mathrm{B}$ and $\mathrm{F}$ had little effect (model L). The HR for OPV was 0.98 $(P=0.94)$.

Further stratification by the trial intervention, placebo or vitamin $\mathrm{A}$, gave the results in models $\mathrm{M}$ and $\mathrm{N}$ (placebo) and $\mathrm{O}$ and $\mathrm{P}$ (vitamin $\mathrm{A}$ ). In placebo recipients, the pattern was similar to that seen in the overall models $\mathrm{J}$ and $\mathrm{K}$, with virtually no interaction (with $P=0.81$ ) between BCG and DTP for the males, and a strong interaction (with $P=0.003$ ) for the females. Among the female vitamin A recipients, however, this interaction decreased in statistical significance $(P=0.19)$, and the point estimates for DTP receipt or not in the presence of BCG were similar, with HR of 0.45 and 0.28 , respectively. There was a statistically significant interaction for male vitamin A recipients, mainly because of not having had any deaths among those few who received DTP but not BCG, but the estimates for DTP receipt or not among those with BCG were very close, $\mathrm{HR}=0.65$ and 0.52 , respectively. Only among the female placebo recipients was the risk associated with receipt of both BCG and DTP appreciably greater than receipt of only one $(\mathrm{HR}=4.5, P=0.003)$. Comparing the HR for receipt of both DTP and BCG to receipt of neither among females in the vitamin A group $(\mathrm{HR}=0.45)$ to that of females in the placebo group $(\mathrm{HR}=1.2)$, there was an estimated $(1-0.45 /$ $1.2) \times 100 \%=63 \%$ reduction in the relative hazard $(P=0.09)$. Similar results were obtained when models $\mathrm{O}$ and $\mathrm{P}$ were further subdivided according to whether the infant had low birthweight $(<2500 \mathrm{~g})$ or not.

\section{Discussion}

The Tamil Nadu study population, with its moderate levels of immunization coverage coupled with relatively high mortality and intensive follow-up activities, provided an excellent opportunity to investigate potential non-specific associations between infant immunization and early mortality. We were unable, however, to investigate the relationships between measles vaccine and other vaccines and mortality up to 2 years of age, which were part of the hypotheses set forth at the WHO workshop.

All vaccines studied, BCG, DTP, and OPV, were associated with protection in univariate analyses. Much of this effect may be due to confounding variables that are associated both with the propensity to vaccinate and infant care practices or exposure to pathogens. Although our 


\section{H. Moulton et al. Infant immunizations and early infant mortality in India}

Table 4 Regression results of time-varying Cox proportional hazards models of mortality among infants; 1998-2002, Tamil Nadu, India

\begin{tabular}{|c|c|c|c|c|c|}
\hline & Model & Deaths & Hazard ratio & $95 \% \mathrm{CI}$ for $\mathrm{HR}$ & $P$-value \\
\hline A & BCG & 208 & 0.62 & $0.45-0.84$ & 0.003 \\
\hline B & BCG, Adj.* & 208 & 0.72 & $0.52-0.99$ & 0.046 \\
\hline $\mathrm{C}$ & First OPV & 208 & 0.72 & $0.51-1.0$ & 0.052 \\
\hline $\mathrm{D}$ & First OPV, Adj.* & 208 & 0.66 & $0.47-0.93$ & 0.019 \\
\hline $\mathrm{E}$ & First DTP & 208 & 0.70 & $0.45-1.08$ & 0.108 \\
\hline $\mathrm{F}$ & First DTP, Adj.* & 208 & 0.77 & $0.49-1.19$ & 0.240 \\
\hline \multirow[t]{2}{*}{ G } & BCG & 208 & 0.63 & $0.46-0.87$ & 0.005 \\
\hline & DTP & & 0.75 & $0.48-1.18$ & 0.212 \\
\hline \multirow[t]{3}{*}{$\mathrm{H}$} & BCG & 208 & 0.63 & $0.46-0.87$ & 0.005 \\
\hline & OPV & & 0.76 & $0.54-1.1$ & 0.135 \\
\hline & DTP & & 0.85 & $0.53-1.4$ & 0.517 \\
\hline \multirow[t]{4}{*}{ I. } & No DTP, no BCG & 208 & $1.0 \$$ & & \\
\hline & DTP, no BCG & & 0.44 & $0.24-0.79$ & 0.007 \\
\hline & No DTP, BCG & & 0.44 & $0.29-0.66$ & $<0.0001$ \\
\hline & DTP, BCG & & 0.72 & $0.43-1.2$ & 0.198 \\
\hline
\end{tabular}

-Tests of multiplicativity, two vaccines same as one: $P=0.0007, P=0.066$

HR for two $v$ s. one: 1.6 ; $95 \%$ CI: $0.97-2.8$

\begin{tabular}{lllll}
\hline $\mathrm{J}$ & 98 & & \\
Males & $1.0 \ddagger$ & $0.24-1.2$ & 0.150 \\
No DTP, no BCG & 0.54 & $0.33-0.98$ & 0.043 \\
DTP, no BCG & 0.57 & $0.27-1.3$ & 0.198
\end{tabular}

TTests of multiplicativity, two vaccines same as one: $P=0.25, P=0.88$

HR for two $v$ s. one: 1.1 ; $95 \%$ CI: $0.48-2.4$

\begin{tabular}{llll}
\hline $\mathrm{K}$ & 110 & & \\
Females & & $1.0 \ddagger$ & $0.15-0.83$ \\
No DTP, no BCG & 0.35 & $0.17-0.62$ \\
DTP, no BCG & 0.33 & $0.43-1.6$
\end{tabular}

-Tests of multiplicativity, two vaccines same as one: $P=0.0005, P=0.015$

HR for two $v s$. one: $2.4 ; 95 \% \mathrm{CI}: 1.2-5.0$

\begin{tabular}{lllll}
\hline $\mathrm{L}$ & 110 & & & \\
& Females, Adj. $\dagger$ & $1.0 \ddagger$ & $0.15-0.88$ & \\
No DTP, no BCG & & 0.38 & $0.20-0.73$ & 0.031 \\
DTP, no BCG & & 0.38 & 0.003 \\
No DTP, BCG & 0.99 & $0.49-2.08$ & 0.980
\end{tabular}

TTests of multiplicativity, two vaccines same as one: $P=0.0006, P=0.010$

HR for two $v$ s. one: 2.6 ; $95 \%$ CI: $1.3-5.4$

\begin{tabular}{|c|c|c|c|c|c|}
\hline \multirow[t]{5}{*}{ M } & Males, placebo & 58 & & & \\
\hline & No DTP, no BCG & & $1.0 \ddagger$ & & \\
\hline & DTP, no BCG & & 0.90 & $0.36-2.3$ & 0.830 \\
\hline & No DTP, BCG & & 0.54 & $0.26-1.1$ & 0.100 \\
\hline & DTP, BCG & & 0.58 & $0.20-1.7$ & 0.306 \\
\hline \multicolumn{6}{|c|}{$\begin{array}{l}\text { TTests of multiplicativity, two vaccines same as one: } P=0.81, P=0.69 \\
\text { HR for two } v \text { s. one: } 0.81 ; 95 \% \mathrm{CI}: 0.29-2.3\end{array}$} \\
\hline \multirow[t]{3}{*}{$\mathrm{N}$} & Females, placebo & 54 & & & \\
\hline & No DTP, no BCG & & $1.0 \ddagger$ & & \\
\hline & DTP, no BCG & & 0.11 & $0.01-0.83$ & 0.032 \\
\hline
\end{tabular}




\section{H. Moulton et al. Infant immunizations and early infant mortality in India}

Table 4 (Continued)

\begin{tabular}{|c|c|c|c|c|c|}
\hline & Model & Deaths & Hazard ratio & $95 \% \mathrm{CI}$ for $\mathrm{HR}$ & $P$-value \\
\hline & No DTP, BCG & & 0.38 & $0.16-0.91$ & 0.030 \\
\hline & DTP, BCG & & 1.2 & $0.52-2.7$ & 0.690 \\
\hline \multicolumn{6}{|c|}{$\begin{array}{l}\text { TTests of multiplicativity, two vaccines same as one: } P=0.003, P=0.003 \\
\text { HR for two } v \text { s. one: } 4.5 ; 95 \% \text { CI: } 1.7-12\end{array}$} \\
\hline \multirow[t]{5}{*}{$\mathrm{O}$} & Males, vitamin A & 40 & & & \\
\hline & No DTP, no BCG & & $1.0 \ddagger$ & & \\
\hline & DTP, no BCG & & 0.00 & $\mathbb{S}$ & 0.0037 \\
\hline & No DTP, BCG & & 0.52 & $0.25-1.07$ & 0.076 \\
\hline & DTP, BCG & & 0.65 & $0.20-2.2$ & 0.485 \\
\hline \multicolumn{6}{|c|}{$\begin{array}{l}\text { Tests of multiplicativity, two vaccines same as one: } P=0.011, P=0.38 \\
\text { HR for two } v \text { s. one: } 1.8 ; 95 \% \text { CI: } 0.49-6.4\end{array}$} \\
\hline \multirow[t]{5}{*}{$\mathrm{P}$} & Females, vitamin A & 56 & & & \\
\hline & No DTP, no BCG & & $1.0 \ddagger$ & & \\
\hline & DTP, no BCG & & 0.57 & $0.20-1.6$ & 0.277 \\
\hline & No DTP, BCG & & 0.28 & $0.11-0.71$ & 0.008 \\
\hline & DTP, BCG & & 0.45 & $0.14-1.4$ & 0.179 \\
\hline \multicolumn{6}{|c|}{ T Tests of multiplicativity, two vaccines same as one: $P=0.19, P=0.90$} \\
\hline
\end{tabular}

\footnotetext{
* Model includes terms for the characteristics in Table 2, hot (April-June) and wet (July-September) seasons, and vaccination-specific propensity score.

$\uparrow$ Model includes terms for OPV, hot and wet seasons, low birth weight, $\geq 3$ prior live births, and BCG vaccination propensity score.

\pm Reference category.

$\mathbb{S}$ Coefficient $=-\infty$; CI not calculable, $P$-value from likelihood ratio test.

- The first is a standard test of interaction, addressing whether having received both immunizations carries the same risk as the product of the risks associated with receipt of each one separately. The second tests whether the risk associated with having received both immunizations is any different from having received just one, or the other, vaccine. This is carried out by employing only two dummy variables, one for receipt of neither vaccine, the other for receipt of both.
}

adjustments for numerous measures of demographic and economic status resulted in minimal changes, they might not capture important cultural and psychological aspects of caretakers. Thus, we cannot determine if the observed association between early receipt of BCG and mortality reduction is due to a biologic effect or an unidentified selection bias. Breiman et al. (2004) observed similar mortality reductions associated with receipt of BCG or DTP, but not among injury-related deaths, thereby giving weight to the possibility of non-specific beneficial effects.

Among females, DTP was associated with the disappearance of the positive association of BCG receipt, but this was not seen for male infants. BCG receipt was associated with a clear benefit, and DTP receipt was always beneficial in the absence of BCG receipt. Similarly, DTP receipt was beneficial or neutral in the presence of BCG receipt, except for girls who did not receive vitamin $\mathrm{A}$. Vitamin A supplementation at birth appeared to mitigate this interaction in girls. Girls who received vitamin A, BCG, and DTP had a mortality hazard similar to those of girls who received vitamin $\mathrm{A}$ and either of these two vaccines alone (model P). The mechanism through which newborn vitamin A supplementation works to reduce mortality and affect responsiveness to vaccines is not well understood. Two potential explanations include effects on tissue maturation and the immune response. A dose of vitamin A early in the neonatal period may provide a stimulus to more rapid maturation of respiratory and gut epithelium leading to increased resistance to invasion and/ or improved clearance of pathogenic organisms; it can also induce changes in immune response profiles (Pasatiempo et al. 1990; Semba et al. 1993; Cantorna et al. 1994; Ross 1996). The complex relationships involving immune system stimulation through various combinations of vaccines and vitamin A, however, remain to be fully elucidated. Moreover, the study was not designed to be able to distinguish between magnitudes of interactions, which would have required much larger sample sizes; the BCG/ DTP interaction in female placebo recipients, and the one in female vitamin A recipients, was biologically but not statistically significant $(P=0.09)$. These results must therefore be interpreted with great caution.

Our results could be interpreted as supportive of the hypothesis that for girls, receipt of both BCG and DTP 


\section{H. Moulton et al. Infant immunizations and early infant mortality in India}

vaccines alters the immune system maturation in a way that does not occur for recipients of one or the other vaccines in isolation. In addition, our data invite modification of this hypothesis: such interference, if it exists, may be ameliorated by newborn vitamin A supplementation in areas with endemic vitamin A deficiency.

One could propose that DTP administration somehow nullifies a protective effect of BCG, which is an interpretation offered by Fine (2001) of the study by Kristensen et al. (2000). This assumes, however, that much of the mortality reduction associated with BCG receipt is due to biologic effects, not just selection effects. For the girls in our study, this is conceivable, as the mortality risks were about the same whether they had neither vaccine or both; moreover, the observed differences according to vitamin $\mathrm{A}$ supplementation status support some kind of biologic action. Similar timing of vaccine receipt for both genders argues against differential selection effects. The boys' data, however, were consistent with strong selection effects associated with receipt of at least one of the vaccines DTP or BCG, with receipt of the other vaccine not being associated with any additional risk. Moreover, our results could also be interpreted as indicating that those who receive BCG and DTP might have had greater survival had they not received BCG. Thus, these results are only partially supportive of the results from Guinea-Bissau. Our results do not appear to support the hypothesis of Benn et al. (2003) that vitamin A potentiates non-specific effects of vaccines; mortality reduction associated with BCG receipt was unaltered by neonatal vitamin A supplementation at birth. In view of the complexity of our results, and the lack of consistently observed effects across studies, we see no reason to alter current immunization strategies or programs in southern India.

\section{Acknowledgements}

This study was funded in part by the Children's Vaccine Program (Program for Appropriate Technology and Health and the Bill and Melinda Gates Foundation) and the World Health Organization. The VASIN study was supported under Cooperative Agreement No. HRN-A-00-97-0001500 between the Johns Hopkins University and the Office of Health and Nutrition, US Agency for International Development, a grant from the Bill and Melinda Gates Foundation, and Task Force Sight and Life. We thank Luke Mullany for his careful preparation of the analysis data sets. We thank members of a WHO advisory group for useful comments on an earlier draft, and in particular, the suggestion to carry out the tests of double $v$ s. single vaccine receipt.

\section{References}

Aaby P, Samb B, Simondon F, Seck AM, Knudsen K \& Whittle H (1995) Non-specific beneficial effect of measles immunisation: analysis of mortality studies from developing countries. British Medical Journal 311, 481-485.

Aaby P, Samb B, Simondon F et al. (1996) Five year follow-up of morbidity and mortality among recipients of high-titre measles vaccines in Senegal. Vaccine 14, 226-229.

Aaby P, Jensen H, Garly ML, Bale C, Martins C \& Lisse I (2002) Routine vaccinations and child survival in a war situation with high mortality: effect of gender. Vaccine 21, 15-20.

Benn CS, Balé C, Sommerfelt H, Frils H \& Aaby P (2003) Hypothesis: Vitamin A supplementation and childhood mortality: amplification of the non-specific effects of vaccines? International Journal Of Epidemiology 32, 822-828.

Breiman RF, Streatfield PK, Phelan M, Shifa N, Rashid M \& Yunus M (2004) Effect of infant immunisation on childhood mortality in rural Bangladesh: analysis of health and demographic surveillance data. Lancet 364, 2204-2211.

Cantorna MT, Nashold FE \& Hayes CE (1994) In vitamin A deficiency multiple mechanisms establish a regulatory $\mathrm{T}$ helper cell imbalance with excess Th 1 and insufficient Th 2 function. Journal of Immunology 152, 1515-1522.

Fine P (2001) Commentary: an unexpected finding that needs confirmation or rejection. British Medical Journal 321, 7-8.

Kabir Z, Long J, Reddaiah VP, Kevany J \& Kapoor SK (2003) Non-specific effect of measles vaccination on overall child mortality in an area of rural India with high vaccination coverage: a population-based case-control study. Bulletin of the World Health Organziation 81, 244-250.

Kristensen I, Aaby P \& Jensen H (2000) Routine vaccinations and child survival: follow up study in Guinea-Bissau, West Africa. British Medical Journal 321, 1-8.

Pasatiempo AMG, Kinshita M, Taylor CE \& Ross AC (1990) Antibody response in vitamin A depleted rats is impaired after immunization with bacterial polysaccharide or protein antigens. Journal of the Federation of American Societies for Experimental Biology 4, 2518-2527.

Rahmathullah L, Tielsch JM, Thulasiraj RD et al. (2003) Impact of supplementing newborn infants with vitamin A on early infant mortality: a community-based randomised trial in southern India. British Medical Journal 327, 254-259.

Rosenbaum PR \& Rubin DB (1983) The central role of the propensity score in observational studies for causal effects. Biometrika 70, 41-55.

Ross AC (1996) The relationship between immunocompetence and vitamin A status. In: Vitamin A Deficiency: Health, Survival, and Vision (eds A Sommer, JKP West) Oxford University Press, New York.

Semba RD, Muhilal WBJ et al. (1993) Abnormal T-cell subset proportions in vitamin A deficient children. Lancet $341,5-8$.

WHO (2001) Workshop on Child Survival and Routine Immunisation. WHO/HTP/V\&B/VAM, Geneva. 
L. H. Moulton et al. Infant immunizations and early infant mortality in India

\section{Authors}

Lawrence H. Moulton (corresponding author), Neal A. Halsey, Joanne Katz, and James M. Tielsch, Department of International Health, Johns Hopkins Bloomberg School of Public Health, 615 N. Wolfe Street, Rm. E5519, Baltimore, MD 21205-2103, USA. Tel.: +1-410-955-6370; Fax: +1-530-325-6867; E-mail: lmoulton@jhsph.edu,nhalsey@jhsph.edu, jkatz@jhsph.edu, jtielsch@jhsph.edu Lakshmi Rahmathullah, Aravind Centre for Women, Children and Community Health, Madurai, Tamil Nadu, India.

Tel.: +91-452-532-653; Fax: +91-452-530-984; E-mail: healthforfamily@yahoo.co.in

R. D. Thulasiraj, Lions-Aravind Institute for Community Ophthalmology, Madurai, Tamil Nadu, India. E-mail: thulsi@aravind.org 